\title{
Revista Brasileira de Enfermagem REBEn \\ Uso da solução salina para manutenção de acessos venosos em adultos: uma revisão
}

REVISÃO

\author{
Use of saline solutions for the mantenaince of venous catheters in adults: a review \\ Uso de la solución salina para la manutención de sondas venosas en adultos: una revisión
}

\section{Francimar Tinoco de Oliveira}

Mestranda do Programa de Enfermagem da UERJ. Enfermeira Monitora da Educação Continuada do Hospital Pró-Cardíaco. Enfermeira da Unidade de Terapia Intensiva do Hospital Municipal Miguel Couto, Rio de Janeiro, RJ.

\section{Lolita Dopico da Silva}

Doutora. Professora Adjunto e orientadora do Curso de Mestrado da Faculdade de Enfermagem da UERJ, Rio de Janeiro, RJ. Enfermeira do Ministério da Saúde.

Coordenadora do Curso de Especialização de Enfermagem Intensivista da UERJ.

Endereço para contato: Rua Flordelice, 505 casa 1. Condomínio Bosque dos esquilos, Jacarepaguá. CEP: 22753808 Rio de Janeiro - RJ. lolita.dopico@gmail.com

Submissão: 03/05/2006

Aprovação: 12/07/2006

\section{Resumo}

Trata-se de uma revisão bibliográfica sobre a prática da salinização em cateteres venosos periféricos. 0 estudo objetivou analisar os artigos científicos sobre o uso de solução salina na manutenção da permeabilidade de cateteres venosos periféricos em adultos, indexados no MedLine e LILACS no período de 1995 a 2005. A amostra consistiu de 5 artigos os quais foram analisados quanto à procedência e periódico de publicação, ao delineamento do estudo, a amostragem, e ao efeito da solução salina. Dois estudos indicam evidências da salinização, outros dois o oposto e um não é conclusivo. Há pequena produção acerca da temática exigindo-se novas pesquisas para validação do método.

Descritores: Enfermagem; Cateterismo periférico; Cuidados de enfermagem.

\section{ABSTRACT}

This article is a bibliography review about the use of saline solution in peripheral venous catheters. The study aimed at analyzing scientific articles about the use of saline solution for the maintenance of peripheral venous catheters patency in adults, indexed at the MedLine and LILACS databases in the periods between 1995 and 2005. The sample consisted of 5 articles analyzed in publication frequency, saline solution effects, study delineation, and nursing periodic publications. The review concluded that this thematic has a modest production and not conclusive about the efficacy of the saline solution in the maintenance patency of peripheral venous catheters in adults, new studies are needed.

Descriptors: Nursing; Catheterization, peripheral; Nursing care.

\section{RESUMEN}

Es una revisión bibliográfica con el objetivo de analizar los resúmenes de salinización de catéteres venosos en el banco de dados MedLine y LILACS en el período de 1995 a 2005 y la base de datos OVID. La muestra se ha compuesto con cinco artículos que se han analizado a partir de la procedencia $y$ periódico de publicación, el delineamiento del estudio, el tamaño de las muestras y si los resultados son conclusivos o no para evidencias de salinización. Dos estudios indican evidencias del efecto de la salinización en la permeabilidad, otro dos lo contrario y uno no es conclusivo. Se concluye que hay poca producción lo que valida nuevas encuestas.

Descriptores: Enfermería; Cateterismo periférico, Atención de enfermería.

Oliveira FT, Silva LD. Uso da solução salina para manutenção de acessos venoso em adultos: uma revisão. Rev Bras Enferm 2006 nov-dez; 59(6): 787-90.

\section{INTRODUÇÃO}

A terapia intravenosa (IV) é considerada como um importante recurso terapêutico, sendo indicado para a maioria dos pacientes hospitalizados, representando por vezes uma condição prioritária para o seu atendimento.

Entre todas as atividades realizadas pelos profissionais de enfermagem a punção venosa periférica é uma das mais freqüentes, exigindo competência técnica para sua realização, destreza manual e domínio de anatomia e fisiologia ${ }^{1}$. Assim, fatores como habilidade prática, a escolha do dispositivo e da veia adequada, a documentação e avaliação do cuidado com o acesso intravascular devem ser consideradas pelo enfermeiro como resultantes de uma reflexão onde se objetiva o melhor cuidado a ser prestado(1).

Verificando fontes de dados internacionais ${ }^{(2,3)}$, encontramos relatos de instituições hospitalares onde a presença de equipes de enfermeiros especializada em terapia IV é justificada por contribuírem com a 
qualidade do serviço oferecida a população, o que se procura garantir através do constante desenvolvimento de práticas padrão, estabelecidas pela Infusion Nursing Society.

A prática da irrigação (flushing) vem sendo realizada com solução salina ou com solução heparinizada ${ }^{(3,4)}$ com várias finalidades porém as principais são a manutenção da permeabilidade e a prevenção de complicações decorrentes da associação medicamentosa

Anteriormente, a manutenção da permeabilidade de acessos venosos era mantida prioritariamente com soluções heparinizadas ${ }^{4}$. Sabe-se que a heparina inibe a formação de coágulos de fibrina in vitro e in vivo e, que seu efeito anticoagulante é praticamente imediato ${ }^{(5)}$. Dadas essas propriedades, a solução heparinizada pode ser utilizada como agente de manutenção da permeabilidade de dispositivos intravenosos, tendo demonstrado sua eficácia há vários anos ${ }^{(4)}$.

A prática da salinização tem sido pesquisada há alguns anos, apresentando como vantagens à heparinização o baixo custo, ser um procedimento mais simples, além de eliminar a possibilidade de incompatibilidade com as drogas e soluções administradas ${ }^{(4,5)}$

O estudo de Epperson ${ }^{(6)}$, realizado há duas décadas, afirmava não haver diferença significativa na manutenção de CVP seja com solução salina ou heparinizada, no entanto, a bibliografia consultada aponta que os dados referentes à eficácia da salinização ainda são inconclusivos ${ }^{(4)}$.

Diante das justificativas acima, estabelecemos como objetivo desta pesquisa analisar as produções científicas sobre salinização em CVP, em bases eletrônicas de dados primários (MedLine) e secundários (OVID).

\section{MÉTODO}

O objeto da pesquisa foi a produção científica existente nas bases eletrônicas de dados primários, Medline, LILACS (Literatura Latino-Americana e do Caribe em Ciências da Saúde) e bases eletrônicas secundárias, a OVID (7), sobre a salinização em cateteres venosos periféricos (CVP) no período de 1995 a 2005.

O levantamento bibliográfico consistiu da primeira etapa de uma pesquisa que está sendo realizada através do método da Prática Baseada em Evidências, de um projeto de dissertação de Mestrado. Teve como finalidade não só apresentar as justificativas de se pesquisar a prática da salinização em veias periféricas, mas também determinar a magnitude do problema, além das evidências já publicadas a respeito da prática da salinização.

Optou-se pelo acesso aos resumos para a análise dessa produção, pois a maioria dos artigos não estava disponível nas bibliotecas regionais. Entendeuse pertinente a análise dos resumos, uma vez, que o conteúdo da maioria atendia aos objetivos do levantamento.

As seguintes etapas foram seguidas para sua elaboração:a) estabelecimento de critérios de seleção da amostra definindo critérios de inclusão e exclusão dos estudos, b) definição das informações a serem extraídas dos resumos, c) análise dos resultados do conteúdo dos estudos e d) destacar aqueles que fazem uma associação da salinização em CVP com a manutenção da permeabilidade dos acessos venosos.

A metodologia usada para a revisão utilizou os seguintes critérios de seleção: a) resumos redigidos em inglês, espanhol ou português com os resumos disponiveis na base de dados, b) detalhar com clareza a metodologia e a forma de análise dos dados, c) terem sido publicados nos últimos 10 anos (1995-2005), d) se referirem a estudos clínicos realizados em adultos acima de 19 anos, e) se referirem ao uso da solução salina comparada ou não a outro tipo de solução na associação com a permeabilidade de cateteres periféricos.

O levantamento foi realizado entre os meses de maio a julho de 2005. Apesar de se ter definido, no formulário acessado, os critérios identificados, alguns estudos foram excluídos por não atenderem as citadas especificações.

A revisão iniciou através do levantamento de estudos primários indexados no banco de dados National Library of Medicine (MedLine) no período de 1995 a 2005. Esse banco de dados pode ser acessada via o site PUBMED, cujo endereço eletrônico é www.ncbi.nlm.nih.gov, ou via site da Biblioteca Virtual em Saúde, cujo endereço eletrônico é www.bvs.br . No site: www.bvs.br acessou-se o banco de dados Medline entre os anos de 1993 a 2005. Utilizouse o formulário avançado, onde na primeira linha se digitou o descritor de assunto "cateterismo periférico", na segunda o descritor de assunto "solução salina" e na terceira o descritor de assunto "heparina" usando o operador and. Obteve-se o resultado de 26 artigos, que ao serem selecionados de acordo com os critérios estabelecidos forneceram uma amostra de tres estudos

Os três estudos selecionados foram os de Fernandez; Griffiths; Murie ${ }^{(8)}$, o de Niesen et al(9) $\mathrm{e} o$ de Meyer et al ${ }^{(10)}$.

Ainda neste mesmo site, foi acessado o banco de dados LILACS (Literatura Latino-Americana e do Caribe em Ciências da Saúde), sendo novamente utilizado o formulário avançado onde na primeira linha se digitou o descritor de assunto "cateterismo periférico", na segunda o descritor de assunto "infusões parenterais" e na terceira o descritor de assunto "heparina" usando o operador and. Foi obtido o resultado de apenas um estudo que apesar de tratar do tema da manutenção de cateteres venosos periféricos com infusäo intermitente, abordava o uso de água destilada e não da solução salina, sendo por esta razão excluído de nossa seleção.

Logo a seguir no site da PUBMED, www.ncbi.nlm.nih.gov, foi utilizada a procura no banco de dados MedLine ,com os descritores "saline solution and heparin", o que levoua um resultado de 43 estudos. Após serem lidos e submetidos aos critérios de seleção foi selecionado o estudo de Myrianthefs et al(11).

Em virtude do número pequeno de resumos encontrados na Medline, optamos por ampliar a busca usando a base de dados secundária OVID. Esta base é acessada pelo site http://gateway.ovid.com, através de senha pessoal. Nela utilizamos a biblioteca virtual referente à enfermagem que se denomina CINAHL (Cumulative Index to Nursing \& Allied Health Literature). Foram utilizados os descritores "saline solution and peripheral venous catheter", obtendo-se então o resultado de sete estudos.

Destes somente um foi selecionado para a amostra, o de Artiolli et al ${ }^{(12)}$. Os outros seis foram excluídos por não atenderem aos critérios definidos.

Após a leitura e tradução de cada resumo, deu-se início à fase de análise dos mesmos. As informações que foram extraídas de cada artigo foram; país onde o estudo ocorreu, o delineamento do estudo, tamanho da amostra, periódico de publicação e ainda a associação entre o tipo de estudo, população e se apresentava resultados conclusivos ou não para permeabilidade de CVP. Os resultados de cada produção foram apresentados de forma descritiva para facilitar ao leitor a sua compreensão

\section{RESULTADOS E DISCUSSÃO}

No quadro 1 está apresentado de forma esquemática os estudos encontrados, associando o ano, país, autor, periódico, amostra, delineamento do estudo e se o mesmo era conclusivo para a permeabilidade.

O estudo mais recente é o de Myrianthefs et al ${ }^{(11)}$ cujo objetivo foi investigar a epidemiologia das complicações em veias periféricas e avaliar três métodos de manutenção de patência e prevenção de tromboflebites. Um total de 300 pacientes de pós-operatório eletivo ortopédico foram estudados prospectivamente, sendo divididos em 3 grupos. Estes grupos eram o grupo controle, onde os CVPs não recebiam irrigação após administração medicamentosa; o grupo solução salina, onde os CVPs recebiam irrigação com $3 \mathrm{ml}$ de solução salina após cada uso do cateter e o grupo heparina, onde os CVPs eram irrigados com $3 \mathrm{ml}$ de solução salina heparinizada contendo $100 \mathrm{U} / \mathrm{ml}$ após cada uso do cateter.

Os resultados referem que ocorreram complicações em $36 \%$ dos pacientes e a incidência de tromboflebites foi de $8 \%$, sendo que destes $4 \%$ no grupo controle. No grupo da solução salina, houve um aumento significativo no total de complicações e obstruções juntamente com tromboflebites quando comparado ao grupo controle. A curva de Kaplan-Meier demonstrou que o grupo controle teve uma elevação significativa na proporção de cateteres sem complicações. O estudo conclui que o uso de solução salina para a manutenção 
Uso da solução salina para manutenção de acessos venoso em adultos: uma revisão

\begin{tabular}{|c|c|c|c|c|c|c|c|}
\hline \multirow{2}{*}{ Ano } & \multirow{2}{*}{ País } & \multirow{2}{*}{ Autores } & \multirow{2}{*}{ Periódico } & \multirow{2}{*}{ Amostra } & \multirow{2}{*}{ Delineamento do estudo } & \multicolumn{2}{|c|}{ Conclusivo para permeabilidade } \\
\hline & & & & & & Sim & Não \\
\hline 2005 & $\begin{array}{l}\text { Greci } \\
a\end{array}$ & $\begin{array}{l}\text { Myrianthefs P; Sifaki M; } \\
\text { Sâmara I; Baltopoulos G }\end{array}$ & J Eval Clin Pract. & \begin{tabular}{|ll}
300 & pacientes \\
ortopédicos &
\end{tabular} & $\begin{array}{l}\text { Prospectivo, observacional com } \\
\text { modelo de intervenção, } \\
\text { randomizado }\end{array}$ & & $x$ \\
\hline 2004 & Italia & $\begin{array}{l}\text { Artiolli G; Finotto S; Lince } \\
\text { MP; Camellini R }\end{array}$ & $\begin{array}{l}\text { Professioni } \\
\text { Infermieristiche }\end{array}$ & 83 pacientes & $\begin{array}{l}\text { Prospectivo, observacional com } \\
\text { modelo de intervenção, } \\
\text { randomizado }\end{array}$ & $x$ & \\
\hline 2003 & EUA & $\begin{array}{l}\text { Niesen KM; Harris DY; } \\
\text { Parkin LS; Henn LT }\end{array}$ & $\begin{array}{l}\text { J Obstet Gynecol } \\
\text { Neonatal Nurs }\end{array}$ & 73 gestantes & $\begin{array}{l}\text { Prospectivo, observacional com } \\
\text { modelo de intervenção, } \\
\text { randomizado, duplo-cego }\end{array}$ & $\mathrm{x}$ & \\
\hline 2003 & $\begin{array}{l}\text { Austrá } \\
\text { lia }\end{array}$ & $\begin{array}{l}\text { Fernandez RS; Griffiths } \\
\text { RD; Murie P }\end{array}$ & $J$ Infus Nurs & $\mathrm{SI}$ & Estudo Survey & si & si \\
\hline 1995 & EUA & $\begin{array}{l}\text { Meyer BA; Little CJ; } \\
\text { Thorp JA; Cohen GR; } \\
\text { Yeast JD }\end{array}$ & Obstet Gynecol & 64 gestantes & $\begin{array}{l}\text { Prospectivo, observacional com } \\
\text { modelo de intervenção, } \\
\text { randomizado, duplo-cego }\end{array}$ & & $x$ \\
\hline
\end{tabular}

Sl= sem informação

Quadro1. Estudos analisados sobre salinização em cateter periférico, 1995-2005.

do cateter em pacientes em pós-operatório ortopédico deve ser evitado. Em pacientes que estejam recebendo heparina de baixo peso molecular, irrigação intravenosa deve ser utilizada para prevenir a obstrução ou tromboflebite na intenção de reduzir os custos e o trabalho de enfermagem.

O estudo de Artiolli et al ${ }^{(12)}$ abordou a comparação do efeito da irrigação do CVP com solução salina antes da oclusão e a cada 6 horas, com outro que utilizou obturador após a suspensão da terapia de infusão. A comparação avaliava a manutenção da permeabilidade e do aparecimento de complicações.

O estudo incluiu 83 pacientes, utilizando a técnica da randomização. Quarenta pacientes foram incluídos no grupo da irrigação e os outros quarenta e três no grupo do obturador. Os resultados do estudo afirmam que não houve diferença significativa na manutenção da permeabilidade e no aparecimento de complicações, entre o grupo que recebeu irrigação com solução salina antes da oclusão do CVP com lavagens a cada 6 horas e com a oclusão do CVP com um obturador sem a irrigação, na suspensão na terapia de infusão.

Oestudo de autoria de Niesen et $a^{(9)}$ é um estudo prospectivo, randomizado, duplo-cego, que teve como objetivo comparar a eficácia entre duas soluções utilizadas para a manutenção de patência em acessos venosos periféricos em gestantes. As soluções avaliadas foram: solução heparinizada $10 \mathrm{UI} / 1 \mathrm{ml}$ versus solução salina $(1 \mathrm{ml})$. As pacientes elegíveis à pesquisa eram aquelas que recebiam a inserção de um CVP para infusão intermitente, as quais eram randomizadas para receber irrigações com solução salina ou heparinizada. Os cateteres recebiam irrigações após cada administração medicamentosa, ou no mínimo a cada 24 horas com a solução determinada (duplo-cego). Todos os sítios também eram avaliados a cada 12 horas para o desenvolvimento de flebites. Aamostra de conveniência foi constituída de 73 gestantes hospitalizadas, entre a 24 e 42 semanas de gestação. Foram excluídas do estudo aquelas com anormalidades na condição cardíaca fetal, dilatação cervical maior de 4 $\mathrm{cm}$, presença de hipersensibilidade a heparina, alterações na coagulação e uso de terapia de anticoagulação (incluindo-se baixas doses de aspirina). Os dados obtidos no estudo indicaram que não houve diferença estatística significante nos CVPs relacionada à permeabilidade e presença de flebite entre as duas soluções. Conclui que tanto a solução salina, quanto a solução heparinizada são igualmente eficazes na manutenção da permeabilidade dos acessos, no entanto, ressalta que devido a sua pequena amostra, outros estudos são necessários para determinar a melhor terapia.

O estudo de Fernandez; Griffiths e Murie ${ }^{(8)}$ investigou as práticas institucionais relacionadas a irrigação de CVP, tendo como objetivo identificar as semelhanças e as diferenças referentes ao tipo e volume da solução de irrigação e a freqüência da mesma para manutenção da permeabilidade. Foram incluídos no estudo 83 hospitais públicos e privados em Sydney, Austrália. O estudo teve o desenho tipo Survey. Buscou relacionar a manutenção da permeabilidade dos CVPs que recebiam irrigação quanto ao tipo, volume e freqüência desta irrigação. Dos 83 hospitais, 11 foram excluídos, 9 por se tratarem de hospitais psiquiátricos e não utilizarem CVP e, dois estavam fechados. Destes 72 hospitais inicialmente incluídos, 5 indicaram que não estavam realizando irrigações em CVP para manutenção da patência. Então, apenas 67 hospitais foram analisados, obtendo-se os seguintes dados: Quanto ao tipo de solução, a mais utilizada foi à solução salina em $96 \%$ nos hospitais públicos (27/28) e em $89 \%$ nos hospitais privados (39/44). A solução heparinizada foi utilizada em apenas um hospital privado representando $4 \%$ (1/28) e, cinco hospitais públicos relataram não praticar a irrigação de CVP correspondendo a $11 \%$ da amostra (5/44).

Quanto ao volume da solução de irrigação, este variou entre as instituições. Volumes inferiores a $5 \mathrm{ml}$ eram utilizados em 5 hospitais, 4 hospitais públicos e 1 hospital privado. Volumes de $5 \mathrm{ml}$ foram usados por 17 hospitais públicos e por 24 hospitais privados. Volumes de $10 \mathrm{ml}$ foram usados por 13 hospitais públicos e por 3 hospitais privados. Cinco hospitais públicos não tinham informações precisas quanto ao volume da irrigação.

Sobre a freqüência das irrigações, 30 hospitais (17 públicos e 13 privados) realizavam as irrigações a cada 4 horas, 18 hospitais (10 públicos e 8 privados) realizavam um intervalo de 6 horas, 4 hospitais ( 2 públicos e 2 privados) mantinham um intervalo de 8 horas entre as irrigações. Respostas obtidas de 15 hospitais (10 públicos e 5 privados) indicavam que suas práticas institucionais orientavam a realização da irrigação apenas quando requisitada.

O estudo não é conclusivo e recomenda que a padronização de práticas baseada nas melhores evidências é extremamente necessária para guiar os profissionais na provisão do cuidado ao paciente.

O estudo realizado por Meyer et al ${ }^{(10)}$ tem uma proposta muito semelhante ao estudo realizado por Niesen et al ${ }^{(9)}$. Trata-se de um estudo clínico, randomizado, duplo-cego, também comparando a solução de heparina e a solução salina na manutenção da permeabilidade do cateter venoso periférico de uso intermitente, em mulheres grávidas entre a 26-34 semanas de gestação, que requisitavam flebotomias constantes. Era realizada a randomização do tipo de solução que seria utilizada para irrigação, solução de heparina ou solução salina, administradas em modo duplo-cego. Os sítios dos cateteres eram acompanhados e as irrigações com a solução randomizada ocorria no mínimo uma vez a cada 6 horas. O Tempo Parcial de Tromboplastina (PTT), era mensurado na inserção do cateter e 48 horas após.

O trabalho concluiu que houve um aumento significativo da permeabilidade dos cateteres no grupo tratado com irrigação de heparina, em 48 a 72 horas (26 de 31 versus 17 de 33 , e 21 de 31 versus 9 de 33 , respectivamente; $p<0.1$ ). Também houve uma significativa redução na freqüência das complicações no cateter neste mesmo grupo ( 4 de 31 versus 13 de $33 ; p<0.1$ ). Não houveram diferenças nos PTTs. O estudo conclui que durante a gestação, o uso de irrigação com heparina para manter a permeabilidade em CVP de uso intermitente resulta em uma excelente razão de manutenção da permeabilidade de 48 a 72 horas após a inserção do cateter, também reduz as taxas de complicações com o cateter e não altera o PTT.

Após a descrição do conteúdo dos resumos, serão apontadas algumas características da produção encontrada.

No que se refere ao período de publicação, dentre os 5 artigos selecionados 
nota-se o predomínio da produção científica sobre salinização em adultos a partir do ano de 2001 , que corresponde a $80 \%$ dos artigos selecionados.

Analisando os resultados expostos, percebe-se que os estudos exibem dados heterogêneos quanto à eficácia da solução salina. De acordo com dois ${ }^{(10,13}$ dos cinco artigos citados, a solução salina apresenta efetividade na manutenção dos CVPs, não apresentando resultados inferiores quando comparada a outro método, nesses casos a heparina. Porém chamamos a atenção ao fato de que no levantamento dessas publicações encontramos populações diferenciadas, comogestantes, pacientes em pós-operatório, pacientes intemados em instituições públicas e privadas. Talvez isto tenha contribuído para um viés de confusão dos resultados o que provavelmente dificulte estabelecer evidencias entre a permeabilidade do CVP e a solução salina.

Reforçando esta impressão, apontamos que Myrianthefs et al ${ }^{(11)}$ recomendam que o uso de solução salina seja evitado na manutenção de CVP de pacientes em pós-operatório por terem verificado uma elevação nas obstruções e tromboflebites. Por outro lado, Meyer et al ${ }^{(10)}$ verificaram superioridade na manutenção da permeabilidade dos cateteres nos grupos que receberam solução com heparina.

O estudo de Niesen et al ${ }^{(9)}$ alerta para a necessidade de novas pesquisas sobre o uso de solução salina na manutenção da permeabilidade dos cateteres venosos, afirmando que os estudos tem utilizado uma amostragem pequena.

Os estudos encontrados também foram analisados quanto à metodologia empregada na sua realização. Ressaltamos o fato de que mesmo se tratando de uma seleção modesta, os estudos incluídos foram realizados com delineamentos de importante impacto metodológico, conforme o quadro 1 explicita.

Ao analisar o desenho metodológico dos estudos selecionados, constatase que das cinco pesquisas, quatro foram estudos prospectivo, observacional com modelo de intervenção e randomizado e destes, apenas dois foram duplo-cego. Sabe-se que estes são os estudos nos quais a determinação da incidência do evento que se avalia é a mais precisa. A randomização é um dos recursos para evitar os diferentes tipos de viés que podem ocorrer nas pesquisas.

Resta destacar que acerca da população envolvida, há um total de 364 pacientes envolvidos nos estudos que não correlacionam a patência do CVP à solução salina. Por outro lado, a população pesquisada nos estudos que dizem haver associação efetiva entre a permeabilidade do CVP e o uso de solução salina, totaliza 156 pessoas.
Não foram encontrados na busca realizada artigos publicados em periódicos de enfermagem nacionais que abordassem a temática deste estudo.

\section{CONSIDERAÇÕES FINAIS}

Apesar de duas décadas após o início das pesquisas nesta área, algumas limitações ainda são atribuídas ao método da salinização, provavelmente por falta de conhecimento e/ou sistematização por parte dos profissionais de enfermagem. É importante ressaltar que muitas instituições do nosso país nem ao menos têm o conhecimento da prática da salinização. No que se refere à pesquisa científica, as limitações desta prática são atribuídas à ausência de um maior número de estudos controlados para validar sua eficácia ${ }^{(6)}$.

Mesmo considerando o fato de que a escolha pela metodologia de pesquisa em bibliotecas virtuais possa ter restringido os resultados obtidos, uma vez que excluímos 06 artigos por falta da apresentação dos resumos, os resultados do estudo mostram que a produção cientifica em grandes bases de dados, no que tange a prática da salinização para manutenção da permeabilidade de CVP em adultos ainda é modesta.

Os resultados encontrados, apesar de limitados a um número pequeno de estudos, expressam que a prática da salinização talvez possa ser considerada uma opção segura na manutenção da permeabilidade dos acessos venosos.

Levando-se em consideração o fato de dois estudos mostrarem-se opostos à realização da salinização de cateteres periféricos e um apresentar-se como inconclusivo, a presença de hipóteses relacionadas à efetividade da solução salina, obstrução e manutenção da permeabilidade do CVP ainda indicam a necessidade de mais estudos nesta temática.

De acordo com o estudo de Pereira e Zanettifi(13) , ainda não há produção científica expressiva no Brasil abordando a terapia IV apesar desta fazer parte de um intenso e cotidiano cuidado de enfermagem. Os estudos dessa natureza com certeza estarão contribuindo com todos aqueles que procuram traçar os resultados do trabalho da enfermagem assim como os seus indicadores de qualidade da assistência ${ }^{(14,15)}$. Concluímos ao afirmar que a prática da terapia IV além de nos conduzir a necessidade de um constante aperfeiçoamento pode ser vista como um campo de pesquisa promissor na demonstração da melhoria dos resultados ligados à prática e às intervenções de enfermagem principalmente em cenários onde a maioria dos pacientes tem um acesso venoso periférico como medida primária de atendimento.

\section{REFERÊNCIAS}

1. Torres $M M$, Andrade $D$, Santos $C B$. Punção venosa periférica: avaliação do desempenho dos profissionais de enfermagem. Rev Latino-am Enfermagem 2005; 13(3): 299-304.

2. Hunter MR. Development of a Vascular Access Team in an acute care setting. J Infus Nurs 2003; 25(2): 85-91.

3. Infusion Nursing Society. Policies and Procedures for infusion nursing. $2^{\text {nd }}$ ed. Norwood (state): INS: 2002.

4. Phillips LD. Manual de terapia intravenosa. $2^{\mathrm{a}}$ ed. Porto Alegre (RS): Artmed; 2001.

5. Rang HP, Dale,M M. Hemostasia e trombose. In: Rang HP, Dale MM. Farmacologia. Rio de Janeiro (RJ): Guanabara-Koogan; 1998. p. 235-47.

6. Epperson EL. Efficacy of $0,9 \%$ sodium chloride injection with and without heparin for maintenance indwelling intermittent injection sites. Clin Pharm 1984; 3(5): 26.

7. Drumond JP, Silva E, Coutinho M. Medicina Baseada em Evidências. Novo paradigma assistencial e pedagógico. Rio de Janeiro (RJ): Atheneu; 2000

8. Fernandez RS, Griffiths RD, Murie P. Peripheral venous catheters: a review of currents practices. J Infus Nurs 2003; 26(6): 388-92.

9. Niesen KM, Harris DY, Parkin LS, Henn LT. The effects of heparin versus normal saline for maintenance of peripheral intravenous locks in pregnant women. J Obstet Gynecol Neonatal Nurs 2003; 32(4): 503-8.

10. Meyer BA, Little CJ, Thorp JA, Cohen GR, Yeast JD. Heparin versus normal saline as a peripheral line flush in maintenance of intermittent intravenous lines in obstetric patients. Obstet Gynecol 1995; 85(3): 433-6.

11. Myrianthefs P, Sifaki M, Sâmara I, Baltopoulos G. The epidemiology of peripheral vein complications: evaluation of the efficiency of differing methods for the maintenance of catheter patency and thrombophlebitis prevention. J Eval Clin Pract 2005; 11(1): 85-8.

12. Artiolli G, Finotto S, Lince MP, Camellini R. A randomized study to manage the peripheral intravenous catheter with saline washing. Professioni Infermieristiche 2004; 57(3): 177-80.

13. Pereira RC, Zanetti ML. Complicações decorrentes da terapia intravenosa em pacientes cirúrgicos. Rev Latino-am. Enfermagem 2000; 8(5): 21-7.

14. Nilce PA, Yoshitome AY. Métodos de avaliação de resultados da assistência de enfermagem. Rev Bras Enferm 2003; 56(1): 8996.

15. Silva LD. Indicadores de qualidade do cuidado de enfermagem na terapia intensiva. Rev Enf UERJ 2003; 11:111-6. 